\title{
Anti-diabetic phytochemicals that promote GLUT4 translocation via AMPK signaling in muscle cells
}

\author{
Kazumi Yagasaki* \\ Division of Applied Biological Chemistry, Institute of Agriculture, Tokyo University of Agriculture and Technology, \\ Fuchu, Tokyo, Japan
}

\begin{abstract}
Skeletal muscles are the largest tissue in our body and play an important role in maintaining glucose homeostasis. Cultured L6 myotubes and $\mathrm{C}_{2} \mathrm{C}_{12}$ myotubes are useful to construct simple glucose uptake assay systems, to screen various phytochemicals that promote glucose uptake, and to clarify their modes of actions. In skeletal muscles, insulin promotes glucose uptake by activating phosphatidylinositol-3 kinase (PI3K) and Akt, leading to increased translocation of glucose transporter 4 (GLUT4) to the plasma membrane. Another GLUT4 translocation promoter is 5' adenosine monophosphate-activated protein kinase (AMPK). In mammalian cells, AMPK activated by an increase in AMP/ATP ratio acts as an energy sensor. AMPK is activated by exercise/contraction in muscle cells and compound such as metformin, this resulting in stimulation of GLUT4 translocation to plasma membrane and hence glucose uptake in skeletal muscles. Thus, studies on novel compounds that activate skeletal muscle glucose uptake and AMPK would be useful for the development of new treatment of insulin resistance and type 2 diabetes (T2D). Here we review the current knowledge of phytochemicals in foods and beverages that promote GLUT4 translocation via AMPK signaling in muscle cells, and their effects on glucose metabolism in mainly T2D model mice. Phytochemicals that have potential to stimulate glucose uptake in muscle cells are suggested to be anti-diabetic.
\end{abstract}

Keywords: Polyphenol, GLUT4, AMPK, muscle, blood glucose, diabetes

\section{Introduction}

The number of diabetic patients is increasing worldwide due to population growth, aging, urbanization, and increasing physical inactivity and prevalence of obesity [1]. The skeletal muscles which account for the majority $(\sim 75 \%)$ of insulin-mediated glucose uptake in the post-prandial state play an important role in maintaining glucose homeostasis [2]. In skeletal muscles, insulin promotes glucose uptake by activating phosphatidylinositol-3 kinase (PI3K) and Akt, leading to increased translocation of glucose trans-

${ }^{*}$ Corresponding author: Kazumi Yagasaki, Ph.D., Division of Applied Biological Chemistry, Institute of Agriculture, Tokyo University of Agriculture and Technology, Fuchu, Tokyo 183-8509, Japan. Fax: +81 42367 5714; E-mail: yagasaki@cc.tuat.ac.jp. porter 4 (GLUT4) to the plasma membrane [2]. Another GLUT4 translocation promoter is $5^{\prime}$ adenosine monophosphate- activated protein kinase (AMPK) which is composed of three subunits [3]. In mammalian cells, AMPK activated by an increase in AMP/ATP ratio acts as an energy sensor [3]. AMPK is activated by exercise/contraction [4] and compounds such as metformin [5], resulting in stimulation of GLUT4 translocation to plasma membrane and hence glucose uptake in skeletal muscles [6]. Thus, studies on novel compounds that activate skeletal muscle glucose uptake and AMPK would be useful for the development of new treatment of insulin resistance and type 2 diabetes (T2D).

We take foods every day to ingest nutrients. Foods also contain non-nutrients such as pigments and condiments, indicating we ingest non-nutrients as well as 
nutrients at the same time. Recently, some food components have been reported to promote glucose uptake and to regulate glucose metabolism. In this article, relationships between food components and glucose metabolism/diabetes will be reviewed, focusing our attention mainly on skeletal muscle cells, AMPK and GLUT4 translocation.

\section{In vitro assay systems for understanding cellular glucose transport}

Leucine, one of the branched-chain amino acids (BCAA), is well known to have insulin-like anabolic effects on protein synthesis [7,8] and degradation [9] in skeletal muscles and cells. In rat skeletal soleus muscles, Nishitani et al. found that L-leucine promoted uptake of $\left[{ }^{14} \mathrm{C}\right] 2$-deoxyglucose, a GLUT4-binding but non-metabolizable analogue of glucose [10]. This assay system is of use to verify effects of food components on glucose uptake, but not necessarily suitable for screening of multi-samples. Thus, we have tried to construct a simple, rapid and low cost assay system for glucose uptake without using radioisotopes by use of a rat skeletal muscle-derived cell line of L6 myoblasts [11] that grow rapidly in culture and differentiate to L6 myotubes (Fig. 1). In this simple assay system, L-leucine was also found to promote glucose uptake, indicating that this assay system with L6 myotubes is comparable to that with rat soleus muscles [10]. Another cell line established from mouse skeletal muscle is $\mathrm{C}_{2} \mathrm{C}_{12}$ myoblasts [12]. Doi et al. reported that L-isoleucine, another BCAA, increased glucose uptake in $\mathrm{C}_{2} \mathrm{C}_{12}$ myotubes by measuring glucose consumption and incorporation of $\left[{ }^{3} \mathrm{H}\right] 2$-deoxyglucose [13]. Thus, L6 myoblasts and $\mathrm{C}_{2} \mathrm{C}_{12}$ myoblasts seem to be useful tools to screen nutrients and non-nutrients that have potential to promote glucose uptake in the skeletal muscles. In addition, these cells are helpful to clarify mechanisms in vitro by which food components promote glucose uptake. Actions of typical foods and their components on glucose metabolism and T2D will be briefly reviewed mainly from the aspects of roles of muscles.

\section{Soy isoflavones and cellular glucose transport}

Genistein and daidzein, soybean isoflavones, have been reported to show anti-diabetic effects in the
T2D model $d b / d b$ mice by enhancing glucose and lipid metabolism [14]. Genistein decreased nonfasting blood glucose levels in KK-Ay/Ta Jcl mice, another T2D animal model [15]. It promoted the translocation of GLUT4 to the cell membrane in L6 myotubes. Isolation of plasma membrane GLUT4 from cytoplasmic GLUT4 was conducted by the procedure of Nishiumi and Ashida [16] with slight modifications [15], using $\mathrm{Na}^{+} / \mathrm{K}^{+}$-ATPase as a marker enzyme of plasma membrane. Based on studies using inhibitors of signaling molecules related to glucose uptake, the stimulatory effect of genistein on glucose uptake appeared to be dependent on the PI3K, mammalian target of rapamycin, protein kinase $\mathrm{C}$ pathway and AMPK pathway. In addition, O-GlcNAcylation by O-(2-acetamido-2-deoxy-D-glucopyranosylidene) amino $\mathrm{N}$-phenyl carbamate, an inhibitor of $\mathrm{N}$ acetylglucosaminidase, reduced the stimulatory effect of genistein on glucose uptake. Taken together, genistein may regulate glucose uptake by increasing the phosphorylation and decreasing the O-GlcNAcylation of proteins related to glucose homeostasis [15]. The translocation of GLUT4 to plasma membrane is often verified by Western blotting analysis (see Fig. 1). Minakawa et al. recently succeeded in construction of HaloTag-glut4 expression vector (pFN21A-rat glut4) and visualizing GLUT4 translocation in L6 myoblasts [17]. We tried to examine whether or not genistein could promote glucose uptake in L6 myoblasts transfected with pFN21A-mock vector or pFN21A-rat glut4 vector (Fig. 2). Glucose uptake was significantly higher in pFN21A-glut4 vector-transfected cells than in pFN21A-mock vector-transfected ones (mock-genistein- vs. glut4-genistein-), suggesting that Halo-GLUT4 derived from transfected pFN21A-glut4 could operate in glucose uptake. Genistein $(50 \mu \mathrm{M})$ significantly promoted glucose uptake in L6 myoblasts transfected with both the pFN21A-mock and pFN21Aglut4 vectors (mock-genistein- vs. mock-genistein+, glut4-genistein- $v s$. glut4-genistein+), indicating that genistein could promote glucose uptake even under the condition of GLUT4 over expression. Caveolin-3 is involved in spatial and temporal regulation of GLUT4 translocation to plasma membrane and hence glucose uptake in skeletal muscle cells [18]. Figure 3 (left) shows cellular localization of Halo-GLUT4 protein, Fig. 3 (centre) shows cellular localization of caveolin3 , and Fig. 3 (right) shows their merging. Genistein $(50 \mu \mathrm{M})$ treatment strengthened co-localization of two proteins in the plasma membrane compartment 


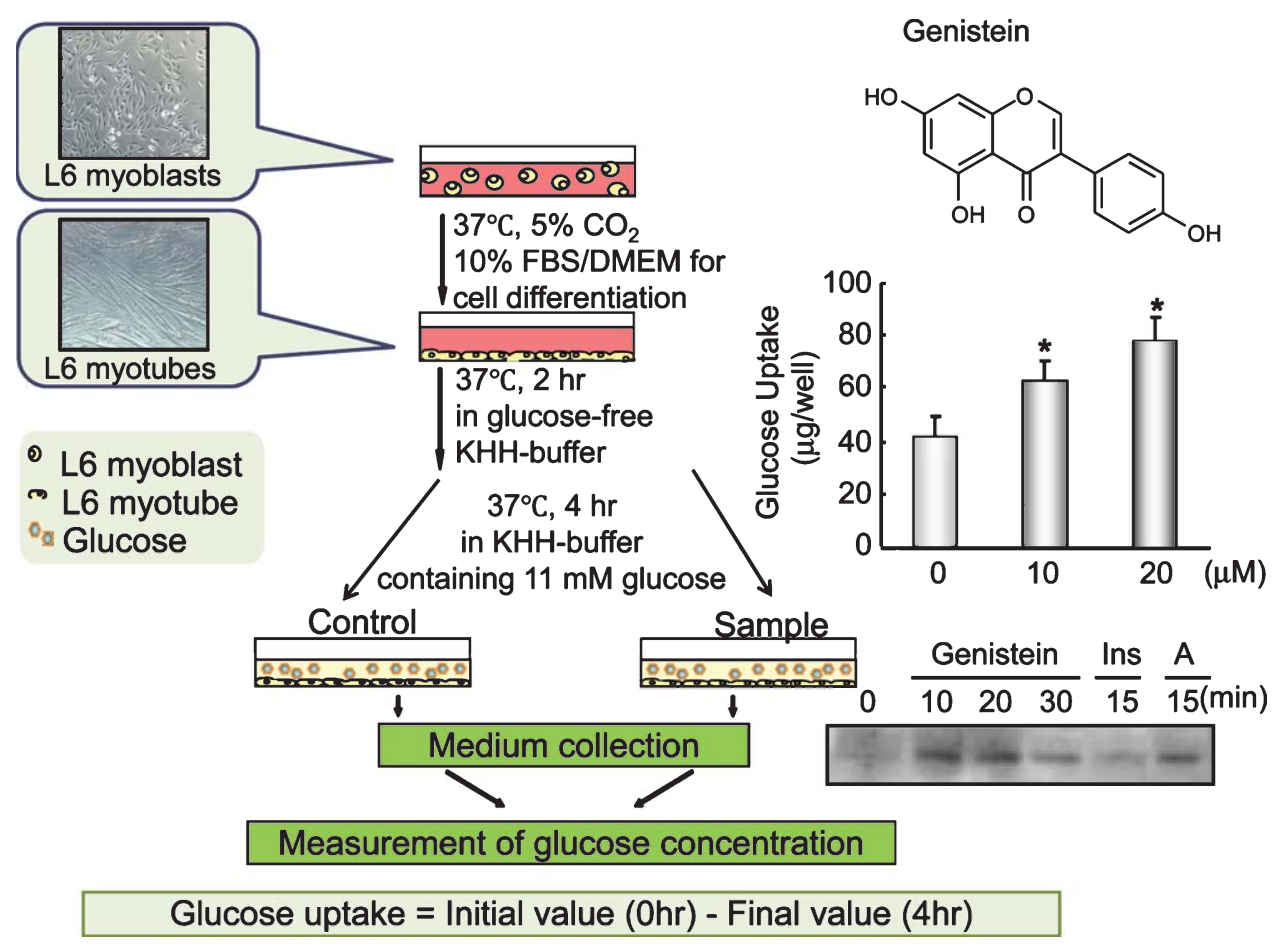

Fig. 1. An example of glucose uptake assay system employing L6 myotubes and effect of genistein on glucose uptake and GLUT4 translocation to plasma membrane (Originally drawn by taking reference 15 into account). L6 myoblasts were cultured and grown for 11 days to form myotubes in 10\% FBS/DMEM. The 11-day-old myotubes were kept for $2 \mathrm{~h}$ in $\mathrm{KHH}$ buffer, and then they were cultured in KHH buffer containing $11 \mathrm{mM}$ glucose without or with samples for appropriate time intervals. Plasma membrane fractions were obtained by the methods described by Nishiumi and Ashida [16] with slight modifications as described previously [15], using $\mathrm{Na}^{+} / \mathrm{K}^{+}$-ATPase as a marker enzyme of plasma membrane. Cell lysate was prepared from the 11-day-old myotubes, and Western blotting for GLUT4, $\mathrm{Na}^{+} / \mathrm{K}^{+}$-ATPase, AMPK, and phospho-AMPK was conducted as described previously [15, 17, 48]. In Fig. 1, a typical result of Western blotting for GLUT4 is shown. Abbreviations: Ins, insulin; A, AICAR (an AMPK activator).

as shown by yellowish colour (glut4-genistein- $v s$. glut4-genistein+). These findings based on bioimaging method support the promoting effect of genistein on GLUT4 translocation to plasma membrane that was demonstrated in biochemical analysis [15].

\section{Polyphenol-rich beverages and cellular glucose transport}

An inverse association between coffee intake and diabetes in humans has been reported [19-24]. Recently, effective coffee components and their modes of actions have been reported. Chlorogenic acid has been reported to stimulate glucose transport in skeletal muscle of $d b / d b$ mice that lack leptin receptor and in cultured L6 myotubes [25]. In L6 myotubes, chlorogenic acid was reported to phosphorylate Akt as well as
AMPK, and these parallel activations in turn increased translocation of GLUT4 to plasma membrane [25]. Since chlorogenic acid is an ester of caffeic and quinic acids, it is interesting to know whether chlorogenic acid and its metabolite, caffeic acid, act directly on skeletal muscle to stimulate AMPK. Caffeic acid, but not chlorogenic acid, has been reported to actually stimulate skeletal muscle AMPK activity and insulinindependent glucose transport with a reduction of the intracellular energy status [26].

Epigallocatechin gallate (EGCG), a tea catechin, is anti-diabetic [27]. A single oral administration of EGCG at $75 \mathrm{mg} / \mathrm{kg}$ body weight promoted GLUT4 translocation in skeletal muscle of rats. EGCG significantly increased glucose uptake accompanying GLUT4 translocation in L6 myotubes at $1 \mathrm{nM}$. The translocation of GLUT4 was also observed both in skeletal muscle of mice and rats ex vivo and in insulin- 


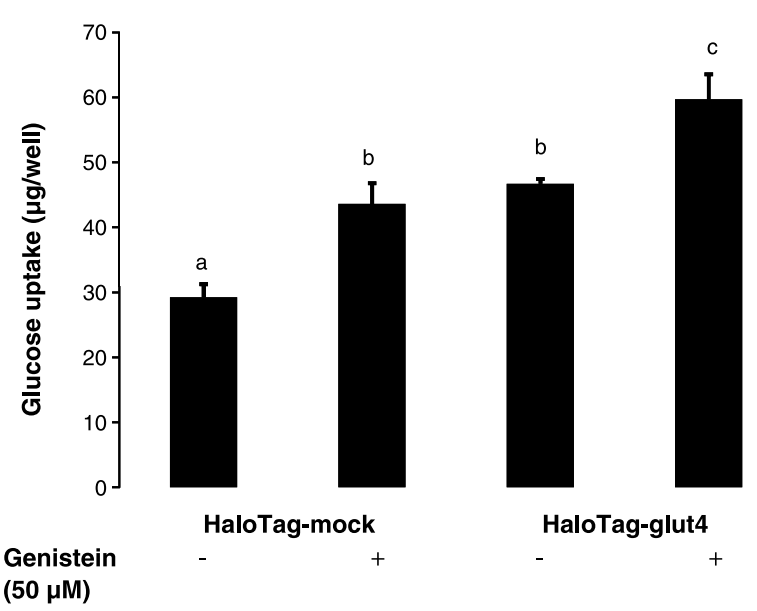

Fig. 2. Effect of genistein on glucose uptake in L6 myoblasts transfected with $\mathrm{pFN} 21 \mathrm{~A}-$ mock vector or pFN21A-glut4 vector. Glucose uptake for $4 \mathrm{~h}$ was measured without or with genistein $(0$, $50 \mu \mathrm{M}$ ) in L6 myoblasts $48 \mathrm{~h}$ after transfection of pFN21A-mock vector or pFN21A-glut4 vector. Each value and bar represents the mean \pm SEM of 4 wells. Values not sharing a common letter are significantly different at $p<0.05$ by Tukey-Kramer multiple comparisons test (Son MJ, Miura Y, Yagasaki K, unpublished data).

resistant L6 myotubes. Therefore, EGCG may improve hyperglycemia by promoting GLUT4 translocation in skeletal muscle with partially different mechanism from insulin. In $d b / d b$ mice, EGCG improved glu- cose tolerance [28]. Thus, dietary supplementation with EGCG could potentially contribute to nutritional strategies for the prevention and treatment of T2D [28]. Increased plasma levels of free fatty acids (FFAs) are associated with profound insulin resistance in skeletal muscle and may also play a critical role in the insulin resistance of obesity and T2D. EGCG and epicatechin gallate (ECG) could improve FFAs-induced insulin resistance and attenuated glucose uptake by activating reduced AMPK and other kinases in $\mathrm{C}_{2} \mathrm{C}_{12}$ skeletal muscle cells, although curcumin showed the best capacity in attenuating insulin resistance [29].

Cacao liquor procyanidin (CLPr) extract, which consisted of $4.3 \%$ catechin, $6.1 \%$ epicatechin, $39.4 \%$ procyanidins and others, ameliorated hyperglycemia and obesity in C57BL/6 mice fed a control or highfat diet for 13weeks [30]. CLPr suppressed high-fat diet-induced hyperglycemia, glucose intolerance and fat accumulation in white adipose tissue. CLPr also promoted translocation of GLUT4 and phosphorylation of AMPK $\alpha$ in the plasma membrane of skeletal muscle and brown adipose tissue. Phosphorylation of $\mathrm{AMPK} \alpha$ was also enhanced in the liver and white adipose tissue [30]. Recently, in a human study, it was shown that polyphenol-rich chocolate attenuated hyperglycemia-induced endothelial dysfunction and oxidative stress [31].
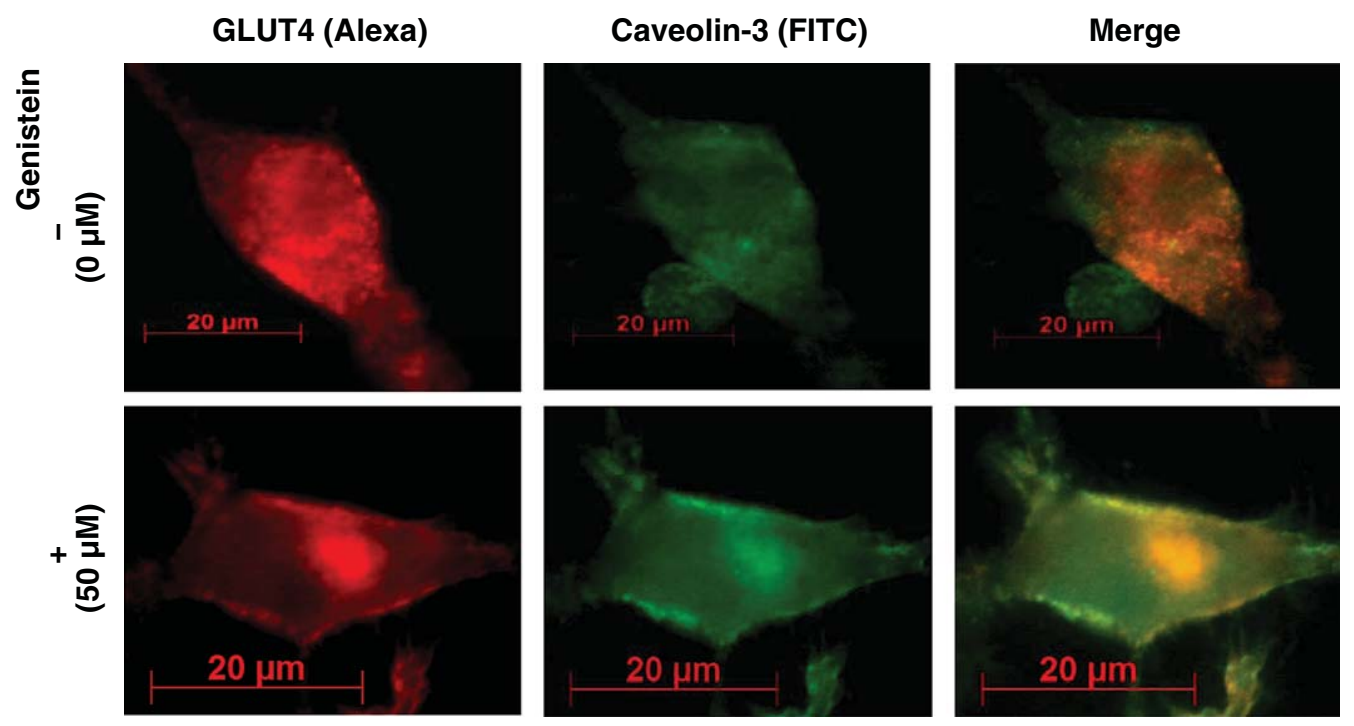

Fig. 3. Effect of genistein on cellular GLUT4 translocalization by bioimaging. L6 myoblasts stably expressing proteins of Halo-GLUT4 were processed for immunocytochemistry using anti-HaloTag antibody and anti-caveolin-3 antibody. Cellular localization of HaloTag-GLUT4 is shown in red fluorescence (left), and that of caveolin-3 is shown in green fluorescence (center). Merged image is also shown (right). L6 myoblasts (genistein-, genistein+) were exposed to genistein $(0,50 \mu \mathrm{M})$ for $30 \mathrm{~min}$ (Son MJ, Miura Y, Yagasaki K, unpublished data). 
As one representative compound of the dihydrochalcone sub-group of polyphenols, aspalathin is only discovered in Rooibos (Aspalathus linearis) which grows exclusively in South Africa. Rooibos contains strong antioxidative substances; in particular aspalathin has higher antioxidative abilities in comparison with other contents [32]. Despite many studies on rooibos and properties of its flavonoids, for example, protective effects against DNA damage [33], inflammation [34], cancer promotion [35], and hyperlipidemia [36], few studies have been attempted to elucidate their anti-diabetic effect. Kawano et al. have reported that aspalathin itself possesses a potential to promote glucose uptake in L6 myotubes and to suppress rises in fasting blood glucose levels and to improve glucose intolerance in T2D model $\mathrm{d} b / \mathrm{d} b$ mice that lack leptin receptor [37]. Aspalathin-enriched green rooibos extract lowered blood glucose levels in streptozotocin (STZ)-induced hyperglycemic rats [38]. Fermented rooibos tea aqueous extract lowered blood glucose levels in diet-induced T2D vervet monkeys [39]. Apalathin has been recently found to suppress the increase in fasting blood glucose levels and improved glucose intolerance in $o b / o b$ mice that lack normal leptin [40]. This polyphenol suppressed the increases in the serum thiobarbituric acid-reactive substances (TBARS), triglyceride (TG) and tumor necrosis factor (TNF)- $\alpha$ levels and the hepatic TG level in $o b / o b$ mice, while it prevented the decrease in serum adiponectin level. Measurement of hepatic gene expression related to glucose and lipid metabolism suggested that aspalathin might reduce, at least partly, hepatic gluconeogenesis and lipogenesis in $o b / o b$ mice. Adiponectin is the most widely studied adipokine in the research of metabolic disease, and experimental and clinical studies have shown that adiponectin improves insulin sensitivity [41]. Adiponectin helps to increase glucose uptake and GLUT4 translocation in rat skeletal muscle cells [42]. AMPK has been reported to be activated by adiponectin in skeletal muscle, where it stimulates glucose uptake and fatty acid oxidation, and inhibits the expression of gluconeogenic genes in the liver [3]. In the study using $o b / o b$ mice, serum adiponectin levels were significantly increased by dietary feeding of aspalathin [40]. This result suggests that aspalathin may, at least partly, play a role in the modulation of insulin sensitivity dependently on adiponectin. Aspalathin was found to activate AMPK and hence to promote GLUT4 translocation to plasma membrane of
L6 myocytes. However, it did not activate Akt phosphorylation. These results indicate that regulation of GLUT4 translocation and hence glucose uptake by aspalathin is due to activation of AMPK but not due to Akt activation. In addition, aspalathin could protect pancreatic $\beta$-cells from advanced glycation end products (AGEs)-induced oxidative stress [40]. Rooibos tea's caffeine-free and comparatively low tannin status, combined with its potential health-promoting properties and most notably antioxidative activity, contributed to its popularity and have promoted studies for potential medicine [39].

\section{Fruit polyphenols and cellular glucose transport}

Resveratrol, a stilbene present in grapes and wine, is well known for its health-promoting properties such as anti-cancer [43-45], anti-hyperlipidemic [46, 47] and anti-nephritic [47] actions. This stilbene was shown to promote glucose uptake at concentrations $1-100 \mu \mathrm{M}$ in L6 myotubes [48]. Resveratrol activated the phosphorylation of AMPK and Akt, and promoted GLUT4 translocation in L6 myotubes, suggesting that resveratrol stimulated glucose uptake and GLUT4 translocation by activating both insulin signalling and AMPK signalling [48]. As mentioned above, aspalathin did not affect Akt phosphorylation, suggesting that target(s) of action(s) may be different among polyphenols. Moreover, resveratrol could protect pancreatic $\beta$-cells in vitro from AGEs-induced oxidative stress, and significantly suppressed the elevation in the serum TBARS levels in $d b / d b$ mice in vivo [48].

Piceatannol, a resveratrol derivative, has been found to decrease the proliferation and invasion of AH109A hepatoma cells in vitro and ex vivo, and to suppress the growth of solid tumors in the hepatoma-transplanted rats [49]. Recently, piceatannol was found to promote glucose uptake through GLUT4 translocation to plasma membrane in L6 myotubes and HaloTagglut4 expression vector-transfected L6 myoblasts in the absence of insulin through AMPK activation, suggesting this stilbene is capable of overcoming insulin resistance. Further, piceatannol suppressed increases in fasting blood glucose concentrations of $d b / d b$ mice 2-3 weeks after oral administration at a dose of $50 \mathrm{mg} / \mathrm{kg}$ body weight and improved glucose intolerance in $d b / d b$ mice given the same dose of piceatannol in intraperitoneal glucose tolerance test (IPGTT) [17]. 
Increasing evidence implies considerable potential of resveratrol to improve health and prevent chronic diseases, but poor bioavailability and rapid metabolism may limit the use of resveratrol in dietary intervention for these diseases [50, 51]. Resveratrol metabolites such as piceatannol may overcome issues of poor bioavailability and rapid metabolism and may be an alternative to resveratrol for the health benefit [51].

Tiliroside contained in several dietary plants, such as strawberry and raspberry, is a glycosidic flavonoid. Recently, the administration of tiliroside to obesediabetic KK-Ay mice has been reported to increase plasma adiponectin levels, to up-regulate the messenger RNA expression levels of adiponectin receptors in the liver and skeletal muscles, and to activate AMPK in both the liver and skeletal muscle with a reduction of plasma insulin levels, with a trend towards a reduction of plasma glucose levels and hence a significant reduction of homeostasis model assessment-insulin resistance (HOMA-IR) [52].

Fermented blueberry juice (BJ) stimulates glucose uptake and AMPK in cultured muscle cells and adipocytes. However, the active principles and their mechanisms of action remain to be identified [53]. Incorporating BJ in drinking water protected young KK-Ay mice against the development of glucose intolerance and diabetes mellitus. The adipokines pathway also seems to be involved because BJ significantly increased adiponectin levels in obese mice [54].

Shabrova et al. analyzed the effects of dietary supplementation with flavonoids extracted from cranberry (FLS) in normal and obese C57/BL6 mice compared to mice maintained on the same diets lacking FLS [55]. Obese mice supplemented with flavonoids showed an amelioration of insulin resistance and plasma lipid profile, and a reduction of visceral fat mass. They have provided evidence that the adiponectin-AMPK pathway is the main mediator of the improvement of these metabolic disorders. In contrast, the reduced plasma atherogenic cholesterol observed in normal mice under FLS seems to be due to a down regulation of the hepatic cholesterol synthesis pathway [55].

\section{Polyphenols in condiment and cellular glucose transport}

Curcumin is a component present in turmeric. It was reported to activate AMPK and to increase glucose uptake in rat L6 myotubes [56]. Recently, its bene- ficial effect has been reported in prediabetic humans [57]. A 9-month curcumin intervention ( $1500 \mathrm{mg} /$ day) in a prediabetic population significantly lowered the number of prediabetic individuals who eventually developed T2D. In addition, the curcumin treatment appeared to improve overall function of $\beta$-cells, with very minor adverse effects. Therefore, this study demonstrated that the curcumin intervention in a prediabetic population may be beneficial [57].

Ginger extract [58] and (S)-[8]-gingerol [59] are recently reported to enhance glucose uptake and GLUT4 translocation in L6 myotubes. We have recently demonstrated beneficial effects of [6]gingerol (GIN) [60]; GIN dose-dependently increased glucose uptake at concentrations of $0-100 \mu \mathrm{M}$ in L6 myotubes and promoted phosphorylation of AMPK, which led to its activation. Judging from co-localization of GLUT4 and caveolin-3, GLUT4 translocation to cellular membrane was enhanced by the addition of GIN. AGEs stimulated reactive oxygen species (ROS) generation in RIN-5F cells, and the treatment of GIN completely abolished the AGEsinduced rise in ROS level in the cells. GIN significantly suppressed the increases in fasting blood glucose levels of $d b / d b$ mice from 1 week after GIN-feeding and thereafter up to 4 weeks. The expression levels of the key gluconeogenic enzymes, phosphoenolpyruvate carboxykinase (PEPCK), glucose-6-phosphatase (G6Pase), glycogen synthase (GS), liver glycogen phosphorylase (LGP) were measured. The expression of both PEPCK and G6Pase was significantly decreased in the GIN group as compared with the control group. Moreover, expression of GS tended to increase and glycogen LGP was decreased by GIN feeding. These findings strongly suggest that GIN has a hypoglycemic effect [60]. Ginger is typical condiment in Asia, and traditionally ingested in many countries. Thus, ginger and its components seem to be useful candidates for prevention of T2D.

Cinnamon is widely used in alternative system of medicine for treatment of diabetes, and cinnamaldehyde was identified as an active principle against diabetes [61]. Cinnamaldehyde has been demonstrated to increase glucose uptake through GLUT4 translocation in skeletal muscle of diabetic rats [61]. Aqueous cinnamon extract and cinnamon polyphenol-enhanced food matrix were effective in lowering fasting blood glucose levels in diet-induced obese hyperglycemic mice, and they decreased gene expression of two major regulators of hepatic gluconeogenesis, PEPCK and 


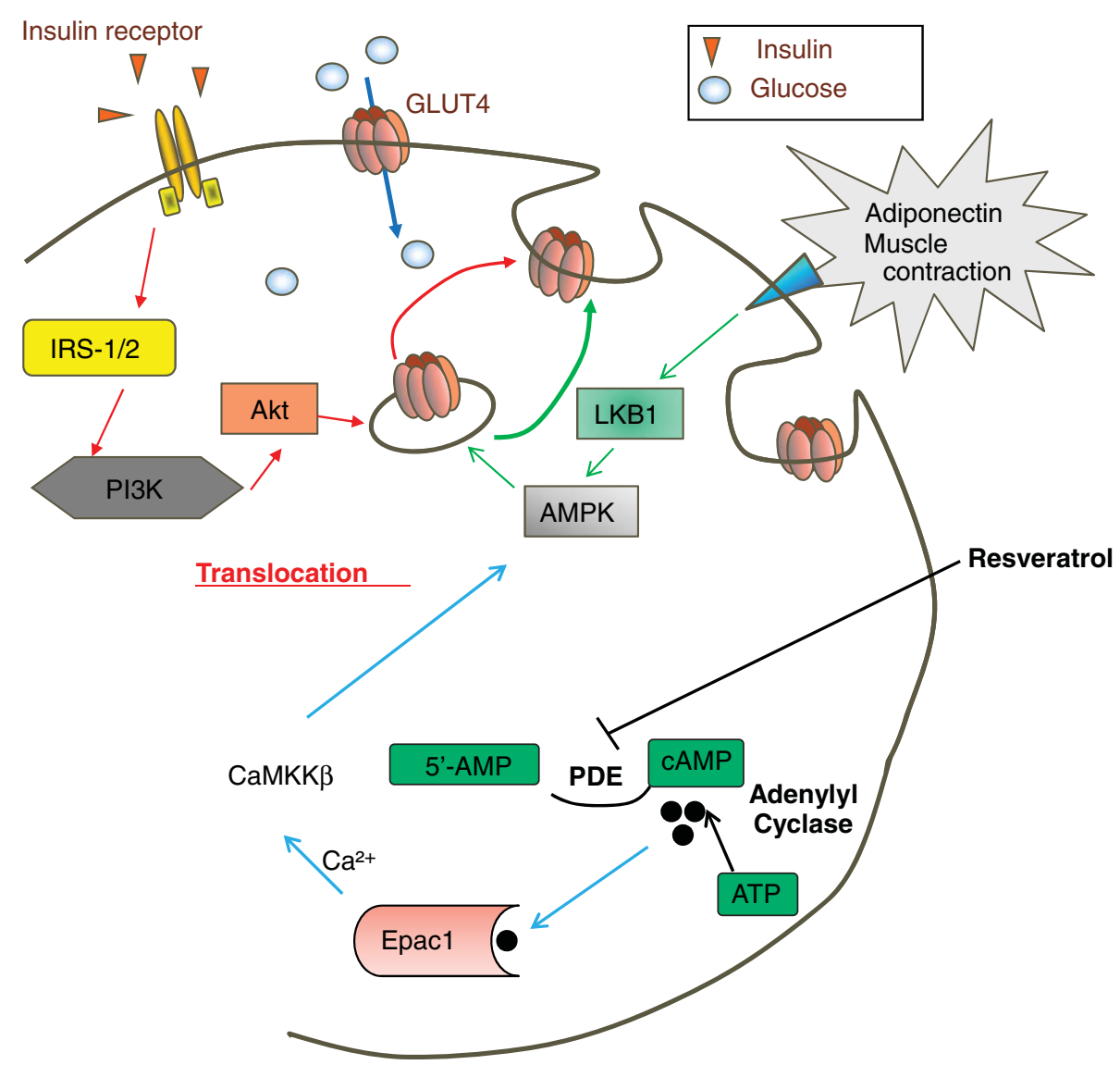

Fig. 4. Schematic representation of signaling pathways leading to GLUT4 translocation through AMPK activation in muscle cells (Originally drawn by taking references 6,64 and 65 into account).

G6Pase [62]. Use of cinnamon showed a beneficial effect on glycemic control in patients with T2D [63].

\section{Inhibition of phosphodiesterases and activation of AMPK}

As above-mentioned, resveratrol was found to activate AMPK in muscle cells. However, the precise mechanism by which resveratrol activates AMPK remains to be elucidated. In particular, the direct molecular target of resveratrol has been elusive [64]. Park et al. recently show that resveratrol directly inhibits cAMP-dependent phosphodiesterases (PDE) [65]. They have reported that the metabolic effects of resveratrol result from competitive inhibition of cAMP-degrading PDE, leading to elevated cAMP levels. The resulting activation of Epac1, a cAMP effector protein, increases intracellular $\mathrm{Ca}^{2+}$ levels and activates the CamKK $\beta$-AMPK pathway via phospholipase $\mathrm{C}$ and the ryanodine receptor $\mathrm{Ca}^{2+}$-release channel. As a consequence, resveratrol increases $\mathrm{NAD}^{+}$and the activity of Sirt1. Inhibiting PDE4 with rolipram reproduces all of the metabolic benefits of resveratrol, including prevention of diet-induced obesity and an increase in mitochondrial function, physical stamina, and glucose tolerance in mice. Therefore, authors suggest that administration of PDE4 inhibitors may also protect against and ameliorate the symptoms of metabolic diseases associated with aging [65]. It is likely that other phytochemicals also activate AMPK by inhibiting PDEs, because many of them have been shown to be PDE inhibitors [66]. Thus, it seems worthy from the aspect of direct target identification to examine whether or not phytochemicals mentioned above could inhibit PDEs (Fig. 4). 


\section{Conclusions}

Many recent studies strongly suggest that food components, namely, non-nutrients such as phytochemicals as well as nutrients that promote glucose uptake in cultured L6 and $\mathrm{C}_{2} \mathrm{C}_{12}$ myotubes seem to be effective in suppressing increased blood glucose concentrations and in improving glucose intolerance in T2D model mice. Promotion of GLUT4 translocation via AMPK activation seems to be a common mechanism by which such polyphenols enhance glucose uptake in L6 myotubes. Thus, the simple, rapid and low cost assay systems with myotubes in culture are suitable for screening many kinds of anti-diabetic phytochemicals.

There have been discrepancies between in vitro and in vivo studies, mainly in effective concentrations of phytochemicals in culture medium and serum/plasma. Some phytochemicals may be absorbable from gastrointestinal tract as such, enter the blood stream, and show their efficacies, while other phytochemicals may be converted by intestinal microbiota to metabolites with biological activity. In some cases, phytochemicals effective in vitro may be metabolized to ineffective forms by intestinal microbiota or in the liver and peripheral tissues after oral ingestion. Thus, it is not easy to determine effective doses of phytochemicals in vivo from in vitro studies. Dose-response studies in vivo both in animals and humans are essential to ascertain whether the effective phytochemicals found in vitro are useful for preventing or delaying disease onset.

In a final message, it is of interest to acknowledge that recently, nutrients have been shown to possess pharmacological actions in addition to serving their expected nutritional roles. The research field studying nutritional roles and metabolism of nutrients in living organisms is called 'nutritional science', and the research field studying mutual interaction between non-nutrients and living organisms has recently been called 'functional food science'. However, the research field investigating pharmacological aspects of nutrients has not been definitely designated. As mentioned above, nutrients as well as non-nutrients possess various pharmacological actions (=food pharmacology). We have proposed to designate food pharmacology as 'bromacology' [67]; 'broma' means 'food', and cology' is a suffix that means 'science' like seen in 'pharmacology'. There is a term "MIBYOU" in Japan. MIBYOU originated from a medical book written in China around 2000 years ago. There is no English word corresponding to MIBYOU. MIBYOU is the transient states between health and disease. Thus, we can say that MIBYOU is semi-health and semi-disease states. Phytochemicals and other food components, by themselves or in combination, have a possibility to prevent the progression of MIBYOU to diagnosed diseases, which is the final aim of bromacology.

\section{References}

[1] Wild S, Roglic G, Green A, Sicree R, King H. Global prevalence of diabetes: Estimates for the year 2000 and projections for 2030. Diabetes Care. 2004;27(5):1047-53.

[2] Saltiel AR, Kahn CR. Insulin signalling and the regulation of glucose and lipid metabolism. Nature. 2001;414(6865):799806.

[3] Towler MC, Hardie DG. AMP-activated protein kinase in metabolic control and insulin signaling. Circ Res. 2007; 100(3):328-41

[4] Hardie DG. AMP-activated protein kinase: A key system mediating metabolic responses to exercise. Med Sci Sports Exerc. 2004;36(1):28-34.

[5] Zou MH, Kirkpatrick SS, Davis BJ, Nelson JS, Wiles WG 4th, Schlattner U, Neumann D, Brownlee M, Freeman MB, Goldman MH. Activation of the AMP-activated protein kinase by the anti-diabetic drug metformin in vivo. Role of mitochondrial reactive nitrogen species. J Biol Chem. 2004;279(42):4394051.

[6] Huang S, Czech MP. The GLUT4 glucose transporter. Cell Metab. 2007;5(4):237-52.

[7] Anthony JC, Yoshizawa F, Anthony TG, Vary TC, Jefferson LS, Kimball SR. Leucine stimulates translation initiation in skeletal muscle of postabsorptive rats via a rapamycin-sensitive pathway. J Nutr. 2000;130(10):2413-9.

[8] Yagasaki K, Morisaki N, Kitahara Y, Miura A, Funabiki $\mathrm{R}$. Involvement of protein kinase $\mathrm{C}$ activation in L-leucineinduced stimulation of protein synthesis in L6 myotubes. Cytotechnology. 2003;43(1-3):97-103.

[9] Nagasawa T, Kido T, Yoshizawa F, Ito Y, Nishizawa N. Rapid suppression of protein degradation in skeletal muscle after oral feeding of leucine in rats. J Nutr Biochem. 2002;13(2):121-7.

[10] Nishitani S, Matsumura T, Fujitani S, Sonaka I, Miura Y, Yagasaki K. Leucine promotes glucose uptake in skeletal muscles of rats. Biochem Biophys Res Commun. 2002;299(5): 693-6.

[11] Yaffe D. Retention of differentiation potentialities during prolonged cultivation of myogenic cells. Proc Natl Acad Sci USA. 1968;61(2):477-83.

[12] Yaffe D, Saxel O. Serial passaging and differentiation of myogenic cells isolated from dystrophic mouse muscle. Nature. 1977;270(5639):725-7.

[13] Doi M, Yamaoka I, Fukunaga T, Nakayama M. Isoleucine, a potent plasma glucose-lowering amino acid, stimulates glucose uptake in $\mathrm{C}_{2} \mathrm{C}_{12}$ myotubes. Biochem Biophys Res Commun. 2003;312(4):1111-7.

[14] Ae Park S, Choi MS, Cho SY, Seo JS, Jung UJ, Kim MJ, Sung MK, Park YB, Lee MK. Genistein and daidzein modu- 
late hepatic glucose and lipid regulating enzyme activities in C57BL/KsJ-db/db mice. Life Sci. 2006;79(12):1207-13.

[15] Ha BG, Nagaoka M, Yonezawa T, Tanabe R, Woo JT, Kato H, Chung UI, Yagasaki K. Regulatory mechanism for the stimulatory action of genistein on glucose uptake in vitro and in vivo. J Nutr Biochem. 2012;23(5):501-9.

[16] Nishiumi S, Ashida H. Rapid preparation of a plasma membrane fraction from adipocytes and muscle cells: Application to detection of translocated glucose transporter 4 on the plasma membrane. Biosci Biotechnol Biochem. 2007;71(9):2343-6.

[17] Minakawa M, Miura Y, Yagasaki K. Piceatannol, a resveratrol derivative, promotes glucose uptake through glucose transporter 4 translocation to plasma membrane in L6 myocytes and suppresses blood glucose levels in type 2 diabetic model db/db mice. Biochem Biophys Res Commun. 2012;422(3): 469-75.

[18] Fecchi K, Volonte D, Hezel MP, Schmeck K, Galbiati F. Spatial and temporal regulation of GLUT4 translocation by flotillin-1 and caveolin-3 in skeletal muscle cells. FASEB J. 2006;20(6):705-7.

[19] van Dam RM, Feskens EJ. Coffee consumption and risk of type 2 diabetes mellitus. Lancet. 2002;360(9344):1477-8.

[20] Isogawa A, Noda M, Takahashi Y, Kadowaki T, Tsugane S. Coffee consumption and risk of type 2 diabetes mellitus. Lancet. 2003;361(9358):703-4.

[21] Yamaji T, Mizoue T, Tabata S, Ogawa S, Yamaguchi K, Shimizu E, Mineshita M, Kono S. Coffee consumption and glucose tolerance status in middle-aged Japanese men. Diabetologia. 2004;47(12):2145-51.

[22] Tuomilehto J, Hu G, Bidel S, Lindström J, Jousilahti P. Coffee consumption and risk of type 2 diabetes mellitus among middle-aged Finnish men and women. JAMA. 2004;291(10): 1213-9.

[23] Carlsson S, Hammar N, Grill V, Kaprio J. Coffee consumption and risk of type 2 diabetes in Finnish twins. Int J Epidemiol. 2004;33(3):616-7.

[24] Agardh EE, Carlsson S, Ahlbom A, Efendic S, Grill V, Hammar N, Hilding A, Ostenson CG. Coffee consumption, type 2 diabetes and impaired glucose tolerance in Swedish men and women. J Intern Med. 2004;255(6):645-52.

[25] Ong KW, Hsu A, Tan BK. Chlorogenic acid stimulates glucose transport in skeletal muscle via AMPK activation: A contributor to the beneficial effects of coffee on diabetes. PLoS One. 2012;7(3):e32718.

[26] Tsuda S, Egawa T, Ma X, Oshima R, Kurogi E, Hayashi T. Coffee polyphenol caffeic acid but not chlorogenic acid increases 5'AMP-activated protein kinase and insulinindependent glucose transport in rat skeletal muscle. J Nutr Biochem. 2012;23(11):1403-9.

[27] Ueda M, Nishiumi S, Nagayasu H, Fukuda I, Yoshida K, Ashida H. Epigallocatechin gallate promotes GLUT4 translocation in skeletal muscle. Biochem Biophys Res Commun. 2008;377(1):286-90.

[28] Ortsäter H, Grankvist N, Wolfram S, Kuehn N, Sjöholm A. Diet supplementation with green tea extract epigallocatechin gallate prevents progression to glucose intolerance in $\mathrm{db} / \mathrm{db}$ mice. Nutr Metab (Lond). 2012;9:11.

[29] Deng YT, Chang TW, Lee MS, Lin JK. Suppression of free fatty acid-induced insulin resistance by phytopolyphenols in
$\mathrm{C}_{2} \mathrm{C}_{12}$ mouse skeletal muscle cells. J Agric Food Chem. 2012;60(4):1059-66.

[30] Yamashita Y, Okabe M, Natsume M, Ashida H. Prevention mechanisms of glucose intolerance and obesity by cacao liquor procyanidin extract in high-fat diet-fed C57BL/6 mice. Arch Biochem Biophys. 2012;527(2):95-104.

[31] Mellor DD, Madden LA, Smith KA, Kilpatrick ES, Atkin SL. High-Polyphenol chocolate reduces endothelial dysfunction and oxidative stress during acute transient hyperglycaemia in Type 2 diabetes: A pilot randomized controlled trial. Diabet Med. 2013;30(4):478-83.

[32] Snijman PW, Joubert E, Ferreira D, Li XC, Ding Y, Green IR, Gelderblom WC. Antioxidant activity of the dihydrochalcones Aspalathin and Nothofagin and their corresponding flavones in relation to other Rooibos (Aspalathus linearis) Flavonoids, Epigallocatechin Gallate, and Trolox. J Agric Food Chem. 2009;57(15):6678-84.

[33] Snijman PW, Swanevelder S, Joubert E, Green IR, Gelderblom WC. The antimutagenic activity of the major flavonoids of rooibos (Aspalathus linearis): Some dose-response effects on mutagen activation-flavonoid interactions. Mutat Res. 2007;631(2):111-23.

[34] Baba H, Ohtsuka Y, Haruna H, Lee T, Nagata S, Maeda M, Yamashiro Y, Shimizu T. Studies of anti-inflammatory effects of Rooibos tea in rats. Pediatr Int. 2009;51(5):700-4.

[35] Marnewick JL, van der Westhuizen FH, Joubert E, Swanevelder S, Swart P, Gelderblom WC. Chemoprotective properties of rooibos (Aspalathus linearis), honeybush (Cyclopia intermedia) herbal and green and black (Camellia sinensis) teas against cancer promotion induced by fumonisin B1 in rat liver. Food Chem Toxicol. 2009;47(1):220-9.

[36] Beltrán-Debón R, Rull A, Rodríguez-Sanabria F, Iswaldi I, Herranz-López M, Aragonès G, Camps J, Alonso-Villaverde C, Menéndez JA, Micol V, Segura-Carretero A, Joven J. Continuous administration of polyphenols from aqueous rooibos (Aspalathus linearis) extract ameliorates dietaryinduced metabolic disturbances in hyperlipidemic mice. Phytomedicine. 2011;18(5):414-24.

[37] Kawano A, Nakamura H, Hata S, Minakawa M, Miura Y, Yagasaki K. Hypoglycemic effect of aspalathin, a rooibos tea component from Aspalathus linearis, in type 2 diabetic model $\mathrm{db} / \mathrm{db}$ mice. Phytomedicine. 2009;16(5):437-43.

[38] Muller CJ, Joubert E, de Beer D, Sanderson M, Malherbe CJ, Fey SJ, Louw J. Acute assessment of an aspalathin-enriched green rooibos (Aspalathus linearis) extract with hypoglycemic potential. Phytomedicine. 2012;20(1):32-9.

[39] Joubert E, de Beer D. Rooibos (Aspalathus linearis) beyond the farm gate: From herbal tea to potential phytopharmaceutical. South African J Botatany. 2011;77(4):869-86.

[40] Son MJ, Minakawa M, Miura Y, Yagasaki K. Aspalathin improves hyperglycemia and glucose intolerance in obese diabetic ob/ob mice. Eur J Nutr. 2013;52(6):1607-19.

[41] Esteve E, Ricart W, Fernández-Real JM. Adipocytokines and insulin resistance: The possible role of lipocalin-2, retinol binding protein-4, and adiponectin. Diabetes Care 2009;32(Suppl 2):362-7

[42] Ceddia RB, Somwar R, Maida A, Fang X, Bikopoulos G, Sweeney G. Globular adiponectin increases GLUT4 translocation and glucose uptake but reduces glycogen syn- 
thesis in rat skeletal muscle cells. Diabetologia 2005;48(1): 132-9.

[43] Kozuki Y, Miura Y, Yagasaki K. Resveratrol suppresses hepatoma cell invasion independently of its anti-proliferative action. Cancer Lett. 2001;167(2):151-6.

[44] Miura D, Miura Y, Yagasaki K. Resveratrol inhibits hepatoma cell invasion by suppressing gene expression of hepatocyte growth factor via its reactive oxygen species-scavenging property. Clin Exp Metastasis. 2004;21(5):445-51.

[45] Miura D, Miura Y, Yagasaki K. Restoration by prostaglandins $E_{2}$ and $F_{2 \alpha}$ of resveratrol-induced suppression of hepatoma cell invasion in culture. Cytotechnology. 2003;43(1-3):155-9.

[46] Miura D, Miura Y, Yagasaki K. Hypolipidemic action of dietary resveratrol, a phytoalexin in grapes and red wine, in hepatomabearing rats. Life Sci. 2003;73(11):1393-400.

[47] Nihei T, Miura Y, Yagasaki K. Inhibitory effect of resveratrol on proteinuria, hypoalbuminemia and hyperlipidemia in nephritic rats. Life Sci. 2001;68(25):2845-52.

[48] Minakawa M, Kawano A, Miura Y, Yagasaki K. Hypoglycemic effect of resveratrol in type 2 diabetic model $\mathrm{db} / \mathrm{db}$ mice and its actions in cultured L6 myotubes and RIN-5F pancreatic $\beta$-cells. J Clin Biochem Nutr. 2011;48(3):237-44.

[49] Kita Y, Miura Y, Yagasaki K. Antiproliferative and antiinvasive effect of piceatannol, a polyphenol present in grapes and wine, against hepatoma AH109A cells. J Biomed Biotechnol. 2012;2012:672416.

[50] Smoliga JM, Baur JA, Hausenblas HA. Resveratrol and healtha comprehensive review of human clinical trials. Mol Nutr Food Res. 2011;55(8):1129-41.

[51] Kwon JY, Seo SG, Heo YS, Yue S, Cheng JX, Lee KW, Kim KH. Piceatannol, natural polyphenolic stilbene, inhibits adipogenesis via modulation of mitotic clonal expansion and insulin receptor-dependent insulin signaling in early phase of differentiation. J Biol Chem. 2012;287(14):11566-78.

[52] Goto T, Teraminami A, Lee JY, Ohyama K, Funakoshi K, Kim YI, Hirai S, Uemura T, Yu R, Takahashi N, Kawada T. Tiliroside, a glycosidic flavonoid, ameliorates obesity-induced metabolic disorders via activation of adiponectin signaling followed by enhancement of fatty acid oxidation in liver and skeletal muscle in obese-diabetic mice. J Nutr Biochem. 2012;23(7):768-76.

[53] Vuong T, Martineau LC, Ramassamy C, Matar C, Haddad PS. Fermented Canadian lowbush blueberry juice stimulates glucose uptake and AMP-activated protein kinase in insulinsensitive cultured muscle cells and adipocytes. Can J Physiol Pharmacol. 2007;85(9):956-65.

[54] Vuong T, Benhaddou-Andaloussi A, Brault A, Harbilas D, Martineau LC, Vallerand D, Ramassamy C, Matar C, Haddad PS. Antiobesity and antidiabetic effects of biotransformed blueberry juice in KK-Ay mice. Int J Obes (Lond). 2009; 33(10):1166-73.
[55] Shabrova EV, Tarnopolsky O, Singh AP, Plutzky J, Vorsa N, Quadro L. Insights into the molecular mechanisms of the antiatherogenic actions of flavonoids in normal and obese mice. PLoS One. 2011;6(10):e24634.

[56] Kim JH, Park JM, Kim EK, Lee JO, Lee SK, Jung JH, You GY, Park SH, Suh PG, Kim HS. Curcumin stimulates glucose uptake through AMPK-p38 MAPK pathways in L6 myotube cells. J Cell Physiol. 2010;223(3):771-8.

[57] Chuengsamarn S, Rattanamongkolgul S, Luechapudiporn R, Phisalaphong C, Jirawatnotai S. Curcumin extract for prevention of type 2 diabetes. Diabetes Care. 2012;35(11):2121-7.

[58] Rani MP, Krishna MS, Padmakumari KP, Raghu KG, Sundaresan A. Zingiber officinale extract exhibits antidiabetic potential via modulating glucose uptake, protein glycation and inhibiting adipocyte differentiation: An in vitro study. J Sci Food Agric. 2012;92(9):1948-55.

[59] Li Y, Tran VH, Duke CC, Roufogalis BD. Gingerols of zingiber officinale enhance glucose uptake by increasing cell surface GLUT4 in cultured L6 myotubes. Planta Med. 2012;78(14): 1549-55.

[60] Son MJ, Miura Y, Yagasaki K. Hypoglycemic effect of [6]-gingerol and its modes of action in vitro and in vivo. Polyphenols Communications 2012. 2012;1:91-92.

[61] Anand P, Murali KY, Tandon V, Murthy PS, Chandra R. Insulinotropic effect of cinnamaldehyde on transcriptional regulation of pyruvate kinase, phosphoenolpyruvate carboxykinase, and GLUT4 translocation in experimental diabetic rats. Chem Biol Interact. 2010;186(1):72-81.

[62] Cheng DM, Kuhn P, Poulev A, Rojo LE, Lila MA, Raskin I. In vivo and in vitro antidiabetic effects of aqueous cinnamon extract and cinnamon polyphenol-enhanced food matrix. Food Chem. 2012;135(4):2994-3002.

[63] Akilen R, Tsiami A, Devendra D, Robinson N. Cinnamon in glycaemic control: Systematic review and meta analysis. Clin Nutr. 2012;31(5):609-15.

[64] Tennen RI, Michishita-Kioi E, Chua KF. Finding a target for resveratrol. Cell. 2012;148(3):387-9.

[65] Park SJ, Ahmad F, Philp A, Baar K, Williams T, Luo H, Ke H, Rehmann H, Taussig R, Brown AL, Kim MK, Beaven MA, Burgin AB, Manganiello V, Chung JH. Resveratrol ameliorates aging-related metabolic phenotypes by inhibiting cAMP phosphodiesterases. Cell. 2012;148(3):421-33.

[66] Kuppusamy UR, Das NP. Effects of flavonoids on cyclic AMP phosphodiesterase and lipid mobilization in rat adipocytes. Biochem Pharmacol. 1992;44(7):1307-15.

[67] Yagasaki K, Yamazaki M, editors. Bromacology: Pharmacology of Foods and Their Components. Trivandrum: Research Signpost; 2008. 